\title{
Numerical Analysis of Conduction of the Action Potential Across the Purkinje Fibre-Ventricular Muscle Junction
}

\author{
Jue $\mathrm{Li}^{1}$, Henggui Zhang ${ }^{2}$, Mark Boyett ${ }^{1}$ \\ ${ }^{1}$ Cardiovascular Sciences, University of Manchester, UK \\ ${ }^{2}$ School of Physics and Astronomy, University of Manchester, UK
}

\begin{abstract}
Normally, there is little conduction delay $(<0.2 \mathrm{~ms})$ between cells in the heart. However, at the Purkinje fibreventricular muscle junction (PVJ), long conduction delays (5-25 ms) are reported. The PVJ therefore has special conduction properties. In support of this, transitional cells are reported at the PVJ. A $1 D$ model was developed consisting of a string of 49 Purkinje cells, connected to one or two transitional cell, connected to one or more strings of 40 ventricular myocytes (a Purkinje fibre is expected to activate a block of ventricular muscle). Rabbit Purkinje cell and left ventricular myocyte action potential models were used; the Purkinje cell model was also used for the transitional cell. The 1D mono-domain model was used to solve conduction. The diffusion coefficient (D) was set to 0.6 and $0.12 \mathrm{~mm}^{2} / \mathrm{ms}$ for the Purkinje fibre and ventricular muscle to give expected conduction velocities. The effects of (i) the diffusion coefficients between the terminal Purkinje cell and the transitional cell (D1) and between the transitional cell and the first ventricular myocyte (D2) and (ii) the 'load' on the Purkinje fibre (i.e. number of strings of ventricular myocytes) were investigated. The greater D1 (up to $\sim 0.4 \mathrm{~mm}^{2} / \mathrm{ms}$ ), the larger the load that could be supported, although further increase in D1 resulted in little further increase. Also the greater D2 (up to $\sim 0.04 \mathrm{~mm}^{2} / \mathrm{ms}$ ), the larger the load that could be supported, but in this case a further increase in D2 resulted in a decrease in the load that could be supported. The delay in conduction at the PVJ was dependent on D1, D2 and load and increased markedly at the smallest values of D1 and D2 and the highest loads. At a certain point, a small change could lead to large increase in the delay or even conduction failure.
\end{abstract}

\section{Introduction}

Purkinje fibers, part of the heart's conduction system, ensure coordinated efficient contraction of the ventricles. The Purkinje fibre-ventricular muscle junction (PVJ) is the site where the Purkinje fibres connect to the working ventricular muscle. Figure 1 shows the His-Purkinje network in the left ventricle of the rabbit. There are three parts: left bundle branch (LBB), connecting Purkinje fibres and terminal Purkinje fibres. The PVJ is where a terminal Purkinje fibre functionally connects with ventricular muscle.

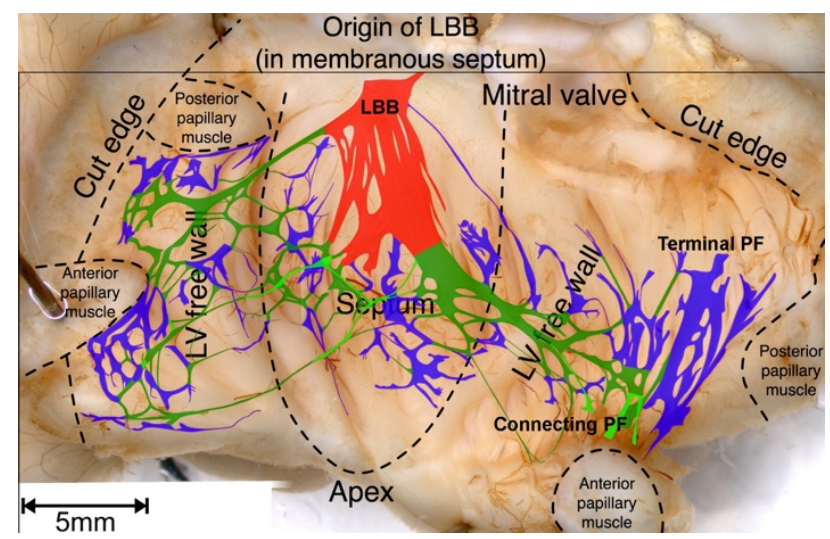

Figure 1. Outline of the His-Purkinje network in the left ventricle of the rabbit. The network has been segmented into different parts - shown in different colours. Red, left bundle branch (LBB); green, connecting Purkinje fibres; blue: terminal Purkinje fibres [1].

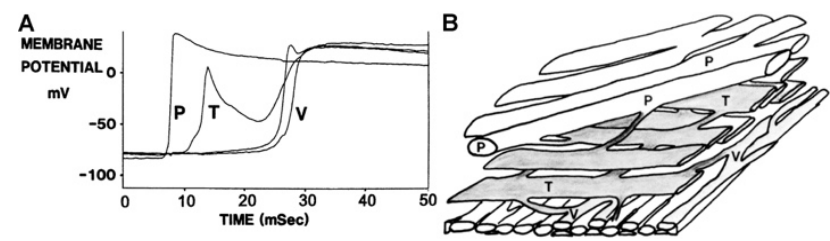

Figure 2. A, action potential upstrokes recorded from a Purkinje cell (P), transitional cell $(\mathrm{T})$ and a ventricular myocyte (V) [2]. B, schematic representation of the structure of a rabbit PVJ [3].

Normally, there is little conduction delay $(<0.2 \mathrm{~ms})$ between cells in the heart. However, at the PVJ, long conduction delays (5-25 ms) [2] and transitional cells have been reported [3]. It has been suggested that there is a relatively high-resistance barrier between Purkinje cells 
(PCs) and transitional cells (TCs) and between TCs and ventricular myocytes (VMs) [3].

In this study, a one-dimensional (1D) model consisting of a Purkinje fibre (made up of PCs), TCs and VMs was used to investigate the effects of the diffusion coefficients between the terminal PC and the TC and between the TC and the first VM on the conduction across the PVJ and the 'load' that can be supported by the Purkinje fibre.

\section{Methods}

\section{1. $1 D$ multicellular model}

The structure of the PVJ is very complex (Figure 2B). In this study, a simplified $1 \mathrm{D}$ multicellular model was developed to investigate factors affecting conduction across the PVJ. The model consisted of a string of 49 PCs, connected to one or more TCs, connected to one or more strings (branches) of 40 VMs. Every TC, as well as every VM branch are the same and arranged in parallel. The cell length is $100 \mu \mathrm{m}$. A Purkinje fibre is expected to activate a block of ventricular muscle. Figure 3 shows the 1D model.

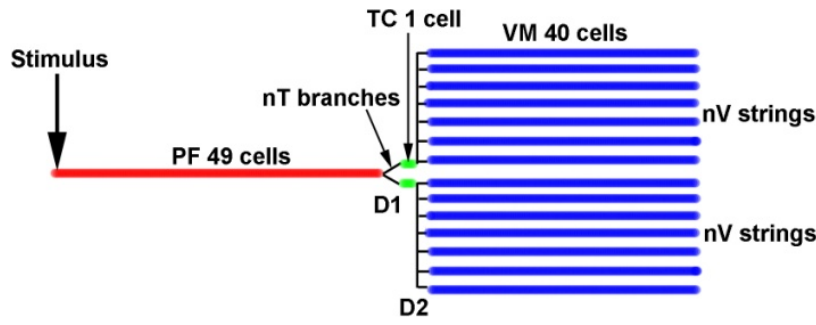

Figure 3. 1D multicellular PVJ model. nT, number of transitional branches; $\mathrm{nV}$, number of $\mathrm{VM}$ branches; $\mathrm{D} 1$, the diffusion coefficient between the terminal PC and the TC; D2, the diffusion coefficient between the TC and the first VM.

1D mono-domain reaction-diffusion model (1) was used to solve the propagation of the action potential:

$$
\frac{\partial V_{m}}{\partial t}=\frac{\partial}{\partial x}\left(D \frac{\partial V_{m}}{\partial x}\right)-\frac{I_{\text {ion }}}{C_{m}}
$$

where $V(\mathrm{mV})$ is the membrane potential, $t(\mathrm{~ms})$ is the time, $I_{\text {ion }}$ (pA) is the total membrane current, $C_{m}(\mathrm{pF})$ is the membrane capacitance, $x$ represents the location of a cell in the 1D model, and $D$ is the diffusion coefficient. $D$ was set to $0.6 \mathrm{~mm}^{2} / \mathrm{ms}$ for the Purkinje fibre and $0.12 \mathrm{~mm}^{2} / \mathrm{ms}$ for ventricular muscle to give expected conduction velocities. The stimulus was applied to first three PCs of the Purkinje fibre string.

For PCs (cells<49) and VMs (cells>51):

$$
\frac{\partial}{\partial x}\left(D \frac{\partial V_{i}}{\partial x}\right)=D \frac{\left(V_{i-1}-2 V_{i}+V_{i+1}\right)}{l_{c}^{2}}
$$

where $l_{c}$ is cell length.
For the PVJ (cell 50 is the TC):

$$
\begin{aligned}
& \frac{\partial}{\partial x}\left(D \frac{\partial V_{49}}{\partial x}\right)=\frac{n T \square D_{1}\left(V_{50}-V_{49}\right)-D_{P F}\left(V_{49}-V_{48}\right)}{l_{c}^{2}} \\
& \frac{\partial}{\partial x}\left(D \frac{\partial V_{50}}{\partial x}\right)=\frac{n V \square D_{2}\left(V_{51}-V_{50}\right)-D_{1}\left(V_{50}-V_{49}\right)}{l_{c}^{2}} \\
& \frac{\partial}{\partial x}\left(D \frac{\partial V_{51}}{\partial x}\right)=\frac{D_{V M}\left(V_{52}-V_{51}\right)-D_{2}\left(V_{51}-V_{50}\right)}{l_{c}^{2}}
\end{aligned}
$$

\subsection{Purkinje cell, transitional cell and ventricular myocyte action potential models}

Healthy rabbit PC and VM action potential models [4] were used. General equations are:

$$
\begin{gathered}
I_{\text {ion }}=I_{N a}+I_{N a L}+I_{C a L}+I_{C a T}+I_{t o}+I_{K r}+ \\
I_{K s}+I_{K 1}+I_{K p}+I_{N a C a}+I_{N a K}+I_{N a, b}+ \\
I_{C a, b}+I_{K, b}+I_{C l}+I_{C l, b}+I_{S L C a, p}
\end{gathered}
$$

There is no TC model available; no patch clamp data exist for single TCs. Hence the PC model was used for the TC as well.

\section{Results}

The diffusion coefficients between the terminal PC and the TC (D1) and between the TC and the first VM (D2), and the number of TC branches (nT) and the number of $\mathrm{VM}$ branches $(\mathrm{nV})$ were investigated.

\subsection{Influence of the diffusion coefficient (D2) between the TC and first VM on the load (nV) that can be supported}

Diffusion coefficients represent the coupling conductance between neighbouring cells. For $n \mathrm{~T}=1$, we investigated the influence of the diffusion coefficient D2 on the load $(\mathrm{nV})$ that could be supported by the Purkinje fibre. $\mathrm{nV}$ is the number of strings of VMs. Because load increases in cardiac tissue are not limited to an integer, $\mathrm{nV}$ can be non-integer and $\mathrm{nV} \geq 1$. We studied $\mathrm{D} 2$ required to support the load $(\mathrm{nV})$ with the action potential propagating successfully from the Purkinje fibre to the ventricular muscle. The minimum D2 is 0.008546 $\mathrm{mm}^{2} / \mathrm{ms}$ which supports only one string of VMs. Figure 4A,B shows the action potential recorded from the terminal PC, TC and first VM. The simulated action potentials in Figure 4A are similar to the action potentials measured experimentally (Figure 2A) [2]. The results show that $\mathrm{nV}$ increases as $\mathrm{D} 2$ increases up to 0.05 $\mathrm{mm}^{2} / \mathrm{ms}$, and then there was no further increase in the load that could be supported (Figure 4C). This means that a Purkinje fibre could drive more ventricular muscle if the 
coupling conductance between the TC cell and first ventricular myocyte is increased. However, once the highest load that can be supported is reached, further increase in the coupling conductance will result in no further increase in the load that could be supported.
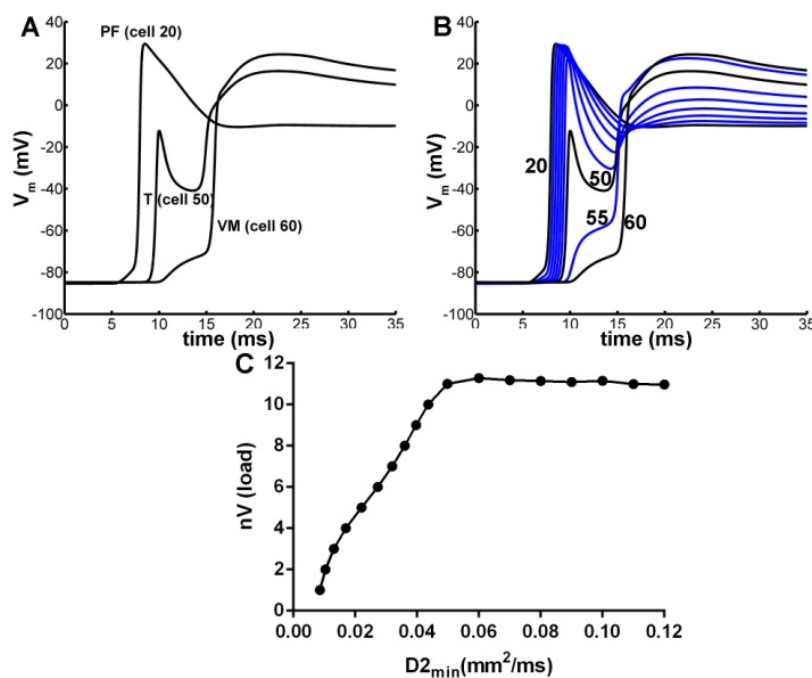

Figure 4. A, action potentials of a PC (cell 20), TC (cell 50) and VM (cell 60); B, action potentials recorded every five cells from the Purkinje fibre (cell 20) to the ventricular muscle (cell 60); C, relationship between load $(\mathrm{nV})$ and minimum D2.

\subsection{Influence of the diffusion coefficients between the terminal PC and the TC (D1) and between the TC and first VM (D2) on the load (nV) that can be supported}

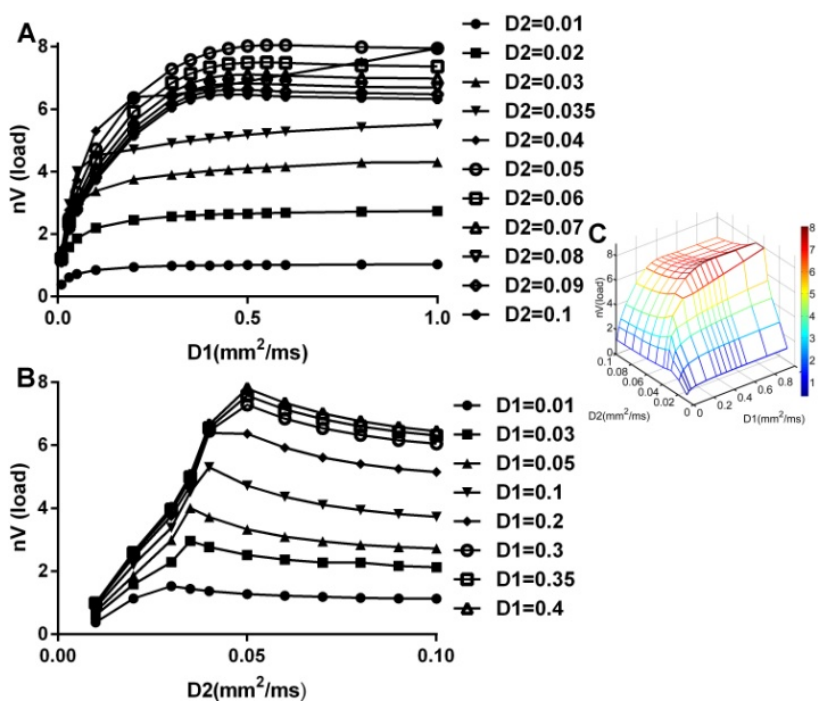

Figure 5. Dependence of the load $(\mathrm{nV})$ that can be supported by the diffusion coefficients D1 (A) and D2 (B). C, a wireframe mesh with colour determined by $\mathrm{nV}$.
For $n T=2$, we investigated that the influence of the diffusion coefficients D1 and D2 on the load (nV) that can be supported. Figure 5 shows the relationship of the load $\mathrm{nV}$ on the diffusion coefficients D1 and D2. The greater D1 (up to $\sim 0.4 \mathrm{~mm}^{2} / \mathrm{ms}$ ), the larger the load that could be supported, although further increase in D1 resulted in little further increase in $\mathrm{nV}$. Also the greater D2 (up to $\sim 0.04 \mathrm{~mm}^{2} / \mathrm{ms}$ ), the larger the load (nV) that could be supported, but in this case a further increase in $\mathrm{D} 2$ resulted in a small decrease in the load that could be supported.

\subsection{The delay in conduction at the PVJ}

The conduction delay at the PVJ was measured between the terminal PC (cell 49) and the second VM (cell 52). We investigated the influence of the diffusion coefficients (D1, D2) and the load (nV) on the delay in conduction at the PVJ. Figure 6 shows one example ( $\mathrm{nT}=2 ; \quad \mathrm{D} 1=0.4 \mathrm{~mm}^{2} / \mathrm{ms} ; \quad \mathrm{D} 2=0.05 \mathrm{~mm}^{2} / \mathrm{ms}$ ): the dependence of the conduction delay at the PVJ on the load $(\mathrm{nV})$. It shows that the delay gradually increases as the load increases until, at the highest load, the propagation of the action potential fails.

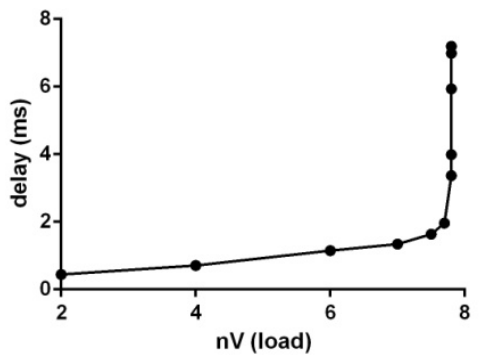

Figure 6. The dependence of the conduction delay at the PVJ on the load $(\mathrm{nV}) . \mathrm{nT}=2 ; \mathrm{D} 1=0.4 \mathrm{~mm}^{2} / \mathrm{ms}$; D2 $=0.05$ $\mathrm{mm}^{2} / \mathrm{ms}$.

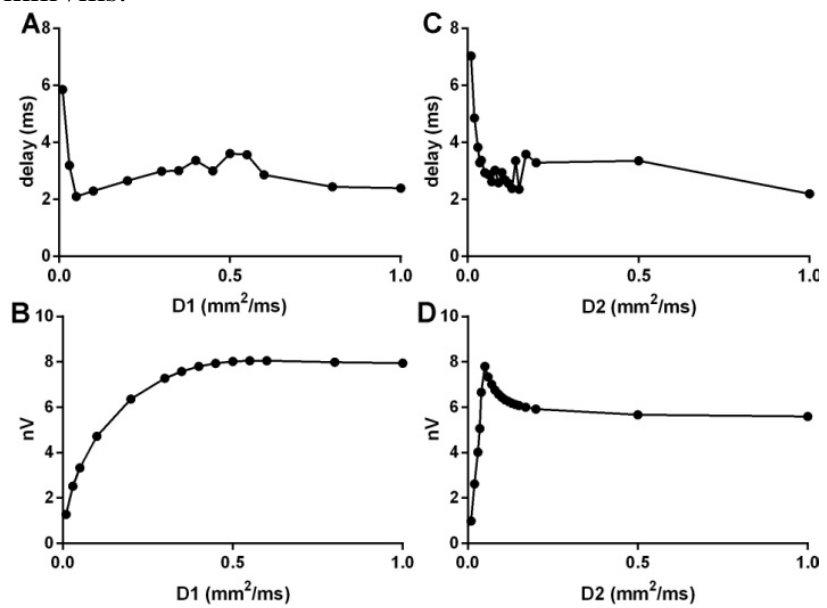

Figure 7. The dependence on the conduction delay at the PVJ on the diffusion coefficients D1 and D2 with the highest loads that could be supported. A and B, D2 $=0.05$ $\mathrm{mm}^{2} / \mathrm{ms}$. C and D, D1 $=0.4 \mathrm{~mm}^{2} / \mathrm{ms}$. $\mathrm{nT}=2$. 
Figure 7 shows that the relationship between the conduction delay at the PVJ and the diffusion coefficients D1 and D2 with the highest loads that could be supported. The results show that the delay increases sharply at smallest diffusion coefficients D1 and D2 before the propagation of the action potential fails.

\subsection{The safety factor for conduction at the PVJ}
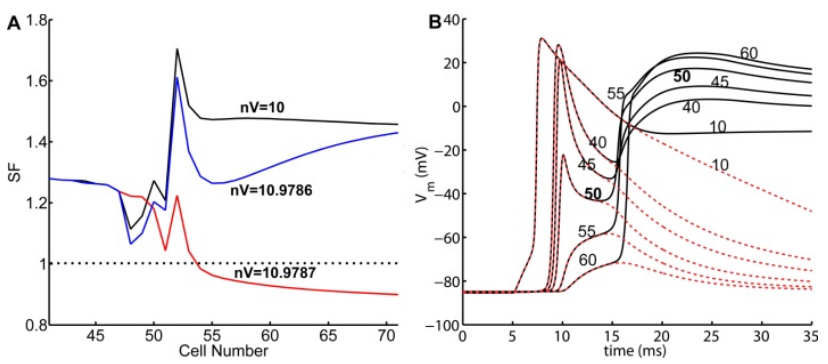

Figure 8. A, the safety factor across the PVJ when the action potential propagates successfully (black and blue traces) and unsuccessfully (red trace); B, action potentials recorded from cells across the PVJ when the action potential propagates successfully (black traces, $\mathrm{nV}=10.9786$ ) and unsuccessfully (red traces, $\mathrm{nV}=10.9788) . \mathrm{nT}=1 ; \mathrm{D} 1=0.6 ; \mathrm{D} 2=0.12$.

The safety factor (SF) for conduction through the PVJ was investigated using the Shaw-Rudy formula [5]:

$$
S F=\frac{\int_{A} I_{c} \llbracket d t+\int_{A} I_{\text {out }} \llbracket d t}{\int_{A} I_{\text {in }} \llbracket d t} A \mid Q_{m}>0
$$

where $I_{c}$ is the capacitive current of the cell, $I_{\text {out }}$ is the current between the cell and its downstream neighbouring cell, and $I_{\text {in }}$ is the axial current that enters the particular cell from its upstream neighbouring cell. $Q_{m}$ is the integral of the current over time. $\mathrm{A}$ is the time period during which the membrane has consumed more charge than it has produced.

Figure 8 shows one example of safety factor and action potentials across the PVJ when the conduction succeeds and fails at the near highest load that could be supported. When $\mathrm{nT}=1, \mathrm{D} 1=0.6$ and $\mathrm{D} 2=0.12$, the conduction fails when $n V>10.9786$. The result shows that the terminal PC (cell 48) had the lowest safety factor when conduction succeeded.

\section{Discussion and conclusion}

A 1D multicellular model consisting of 49 PCs, connected to one or two TCs, connected to one or more strings of 40 VMs (Figure 3) was developed to investigate the influence of the diffusion coefficients D1 and D1 and load $(\mathrm{nV})$ on the PVJ conduction delay. The greater the value of D1 (up to $\sim 0.4 \mathrm{~mm}^{2} / \mathrm{ms}$ ), the larger the load that could be supported, although further increase in D1 beyond $\sim 0.4 \mathrm{~mm}^{2} / \mathrm{ms}$ resulted in little or no further increase in the load that could be supported. Also the greater the value of D2 (up to $\sim 0.04 \mathrm{~mm}^{2} / \mathrm{ms}$ ), the larger the load that could be supported, but in this case a further increase in D2 beyond $\sim 0.04 \mathrm{~mm}^{2} / \mathrm{ms}$ resulted in a decrease in the load that could be supported. The delay in conduction at the PVJ was dependent on both D1 and D2 and increased markedly at the smallest values. The delay was also dependent on the load and increased markedly at the highest loads. At a certain point, a small change in a diffusion coefficient or the load could lead to a large increase in the delay or even conduction failure. Hence, a large increase of the PVJ delay is a risk factor for conduction block.

\section{Acknowledgements}

This study is supported by a British Heart Foundation programme grant (RG/11/18/29257).

\section{References}

[1] Atkinson A, Inada S, Li J, Tellez JO, Yanni J, Sleiman R, Allah EA, Anderson RH, Zhang H, Boyett MR, Dobrzynski $\mathrm{H}$. Anatomical and molecular mapping of the left and right ventricular His-Purkinje conduction networks. J Mol Cell Cardiol 2011;51:689-701..

[2] Wiedmann RT, Tan RC, Joyner RW. Discontinuous conduction at Purkinje-ventricular muscle junction. Am J Physiol Heart Circ Physiol 1996;271:H1507-H1516.

[3] Tranum-Jensen J, Wilde AA, Vermeulen JT, Janse MJ. Morphology of electrophysiologically identified junctions between Purkinje fibers and ventricular muscle in rabbit and pig hearts. Circ Res 1991;69:429-37.

[4] Aslanidi OV, Sleiman RN, Boyett MR., Hancox JC, Zhang $\mathrm{H}$. Ionic mechanisms for electrical heterogeneity between rabbit Purkinje fiber and ventricular cells. Biophys J 2010;98:2420-31.

[5] Shaw RM, Rudy Y. Ionic Mechanisms of Propagation in cardiac tissue: roles of the sodium and L-type calcium currents during reduced excitability and decreased gap junction coupling. Circ Res 1997;81:727-41.

Address for correspondence.

Dr. Jue Li

Division of Cardiovascular Sciences, University of Manchester CTF Building, 46 Grafton Street, Manchester M13 9NT, UK jue.li@manchester.ac.uk 